\title{
Accelerating cosmologies from compactification
}

\author{
Paul K. Townsend* and Mattias N.R. Wohlfarth \\ Department of Applied Mathematics and Theoretical Physics, \\ Centre for Mathematical Sciences, University of Cambridge, Cambridge CB3 0WA, U.K.
}

\begin{abstract}
A solution of the $(4+n)$-dimensional vacuum Einstein equations is found for which spacetime is compactified on an $n$-dimensional compact hyperbolic manifold ( $n \geq 2)$ of time-varying volume to a flat four-dimensional FLRW cosmology undergoing a period of accelerated expansion in Einstein conformal frame. This shows that the 'no-go' theorem forbidding acceleration in 'standard' (time-independent) compactifications of string/M-theory does not apply to 'cosmological' (timedependent) hyperbolic compactifications.
\end{abstract}

PACS numbers: 98.80.Cq, 11.25.Mj, 11.25.Yb, 98.80.Jk

Astronomical observations appear to show that the universe is not only expanding but is undergoing accelerated expansion, see e.g. 1]. In addition, recent measurements of the cosmic microwave background provide support for the hypothesis of accelerated expansion in a much earlier inflationary cosmological epoch, see e.g. 2, 3]. Although it is not difficult to find cosmological models that exhibit these features, one would wish any such model to be derivable from a fundamental, and mathematically consistent, theory that incorporates both gravity and the standard model of particle physics. Most current attempts to place the standard model within such a framework start from the ten or eleven-dimensional spacetime of superstring/M-theory, in which case one needs a compactification of ten or eleven dimensional supergravity in which an effective four-dimensional cosmology undergoes one or more periods of accelerated expansion. However, it has been shown that no such solution exists when the six or seven dimensional 'internal' space is a time-independent non-singular compact manifold without boundary [4, 5]. Three observations go into the derivation of this 'no-go' theorem. The first is that accelerated expansion requires a violation of the strong-energy condition. This is the condition on the stress tensor that, given the Einstein equations, implies $R_{00} \geq 0$, but the acceleration of a FLRW (homogeneous and isotropic) universe is positive if and only if $R_{00}$ is negative. The strong energy condition is violated in many four-dimensional supergravity theories but, and this is the second observation, it is not violated by either elevendimensional supergravity or any of the ten-dimensional supergravity theories that serve as effective field theories for a superstring theory. The third observation is generic to any compactification of the type specified in the theorem: if the higher-dimensional stress tensor satisfies the strong energy condition then so will the lowerdimensional stress tensor.

Clearly, any attempt to derive a viable cosmology from string/M-theory must circumvent this no-go theorem, and this is possible in one of two ways. Either one rejects ten or eleven-dimensional supergravity as the relevant starting point or one relaxes one or more of the premises of the theorem. Attempts to circumvent the theorem by the addition of higher-derivative 'quantum correction' terms to the supergravity action, or appeals to non-geometrical solutions of string theory with no classical analogue would fall into the former category, but we are not aware of any successful attempt along these lines. Here we shall assume that ten or eleven-dimensional supergravity is the relevant starting point. The following options are now available. One can give up the compact condition on the internal space; this has the advantage that there are then known 'compactifications' that allow accelerating four-dimensional cosmologies [6, 7], but the disadvantage that the four-dimensional spectrum is continuous. As no good way around this problem has been found, or seems likely to be found [8], we discard this possibility. The possibility of an internal space that is compact but has a boundary can be considered a special case of an internal space with singular subspaces. This appears to be an attractive way to escape the no-go theorem because M-theory includes branes and boundaries on which the matter fields of the standard model are likely to be located. However, any singular internal space that is the limit of a sequence of non-singular spaces would, by continuity, suffer from the same problems as non-singular internal spaces, so one would expect to have to consider non-resolvable singularities. We are not optimistic about this option, although it certainly deserves a full investigation.

The only remaining option is to give up the condition of time-independence of the internal space. As we are concerned with cosmological solutions, which are intrinsically time-dependent, there is no good reason to suppose that the internal space is not also time dependent. From the four-dimensional perspective this amounts to the supposition that there are time-dependent scalar fields. In this case we need to confront an ambiguity in the choice of 'conformal frame' for the metric: given a scalar field $\phi$ and a metric $g$ one can take

$$
\tilde{g}_{\mu \nu}=e^{2 \phi} g_{\mu \nu}
$$

as a new, conformally rescaled, metric. The choice of conformal frame for which the four-dimensional gravita- 
tional action is of Einstein-Hilbert type, with no scalarfield-dependent multiplicative factor, defines the 'Einstein frame' metric. If one insists on the Einstein conformal frame then it is not immediately obvious how timedependence of the internal manifold helps. Unless the compactification generates a scalar potential, or a cosmological constant, the four-dimensional stress-tensor will still satisfy the strong energy condition. Thus, toroidal compactification (of the vacuum Einstein equations) can never yield an accelerating universe in Einstein frame. This conclusion does not apply if the metric is not in Einstein conformal frame: let $g_{\mu \nu}$ be the four-metric of an FLRW cosmology in standard coordinates, and let the scalar field $\phi$ of (1) depend only on the time coordinate $t$; then

$$
\tilde{R}_{00}=R_{00}-3[\ddot{\phi}(t)+H(t) \dot{\phi}]
$$

where $H(t)$ is the Hubble 'constant'. This shows that positivity of $R_{00}$ does not imply positivity of $\tilde{R}_{00}$.

This point is illustrated by the following Kasner-type metric:

$$
d s^{2}=-d t^{2}+t^{2 \alpha} d \mathbf{x} \cdot d \mathbf{x}+t^{2 \beta} d s^{2}\left(T^{n}\right) .
$$

This solves the $(4+n)$-dimensional vacuum Einstein equations if

$$
\alpha=\frac{3 \pm \sqrt{3 n(n+2)}}{3(n+3)}, \quad \beta=\frac{n \mp \sqrt{3 n(n+2)}}{n(n+3)} .
$$

The upper sign yields a standard decelerating fourdimensional FLRW spacetime. The lower sign yields an accelerating but contracting four-dimensional FLRW spacetime for $n \geq 2$. However, by taking $t \rightarrow\left(t_{\infty}-t\right)$ we get

$$
d s^{2}=d s_{4}^{2}+\left(t_{\infty}-t\right)^{2 \beta} d s^{2}\left(T^{n}\right)
$$

where $d s_{4}^{2}$ is a flat FLRW spacetime with scale factor

$$
a(t)=\left(t_{\infty}-t\right)^{\alpha} .
$$

As $\dot{a}>0$ and $\ddot{a}>0$ for $t<t_{\infty}$ we have accelerated expansion. However $d s_{4}^{2}$ is not the Einstein-frame metric. The Einstein-frame four-metric yields a decelerating universe.

Non-Einstein conformal frames have the feature that the effective Newton constant becomes time-dependent in cosmological solutions. For this reason, among others, what is really needed is a cosmological compactification of ten or eleven-dimensional supergravity that yields a four-dimensional FLRW universe undergoing accelerated expansion in Einstein frame. We have just argued (assuming the absence of a scalar potential generated by fields other than the metric) that this cannot be achieved by any toroidal compactification and the same argument applies to compactification on any Ricci-flat space. However, we shall show, by example, that there exist cosmological compactifications on Einstein spaces of negative curvature that yield accelerating four-dimensional FLRW cosmologies in Einstein frame.

Consider an $n$-dimensional compact Einstein manifold with metric

$$
d \hat{s}_{n}^{2}=\hat{g}_{m n} d y^{m} d y^{n} .
$$

We will take it to have constant negative curvature $\kappa$, so that $n \geq 2$ and

$$
R(\hat{g})_{m n}=-(n-1) \kappa^{2} \hat{g}_{m n} .
$$

Such spaces are obtained by identifying hyperbolic $n$ space under the action of a freely acting discrete subgroup of its $S O(1, n)$ isometry group. The identifications break all continuous isometries, so there will be no massless Kaluza-Klein vector fields resulting from compactification on this space.

Now consider the following $(4+n)$-metric parametrized by functions of time $S(t)$ and $K(t)$ :

$$
d s^{2}=e^{3 n t /(n-1)} K^{-\frac{n}{n-1}} d s_{E}^{2}+e^{-6 t /(n-1)} K^{\frac{2}{n-1}} d \hat{s}_{n}^{2}
$$

where

$$
d s_{E}^{2}=-S^{6} d t^{2}+S^{2} d \mathbf{x} \cdot d \mathbf{x} .
$$

This metric solves the $(4+n)$-dimensional vacuum Einstein equations if

$$
S(t)=e^{-(n+2) t / 2(n-1)} K^{\frac{n}{2(n-1)}},
$$

and

$$
K(t)=\frac{\sqrt{3(n+2) / n}}{(n-1) \kappa \sinh (\sqrt{3(n+2) / n}|t|)} .
$$

Note that $\kappa$ has dimensions of inverse length, but an implicit dimensionful constant appearing in these expressions has been set to unity.

The four-metric $d s_{E}^{2}$ is the Einstein frame metric of the four-dimensional theory that results from the compactification of the $n$ internal dimensions. It takes the standard FLRW form for a flat homogeneous isotropic universe with scale factor $S$ in terms of the time coordinate $\eta$ defined by

$$
d \eta=S^{3}(t) d t .
$$

The four-dimensional universe is expanding if $d S / d \eta>0$. This is equivalent to $m(t)<0$ where

$$
m(t)=1+\sqrt{\frac{3 n}{n+2}} \operatorname{coth}(\sqrt{3(n+2) / n} t) .
$$


The universe undergoes accelerated expansion if, in addition, $d^{2} S / d \eta^{2}>0$. This is equivalent to

$$
m(t)^{2}<\frac{3(n-1) /(n+2)}{\sinh ^{2}(\sqrt{3(n+2) / n} t)} .
$$

Both conditions are satisfied simultaneously for negative $t$ in a certain interval, as can be seen from the plot of $m(t)$ in figure 1 for the $n=7$ case of relevance to M-theory compactifications. It can be shown that the universe is

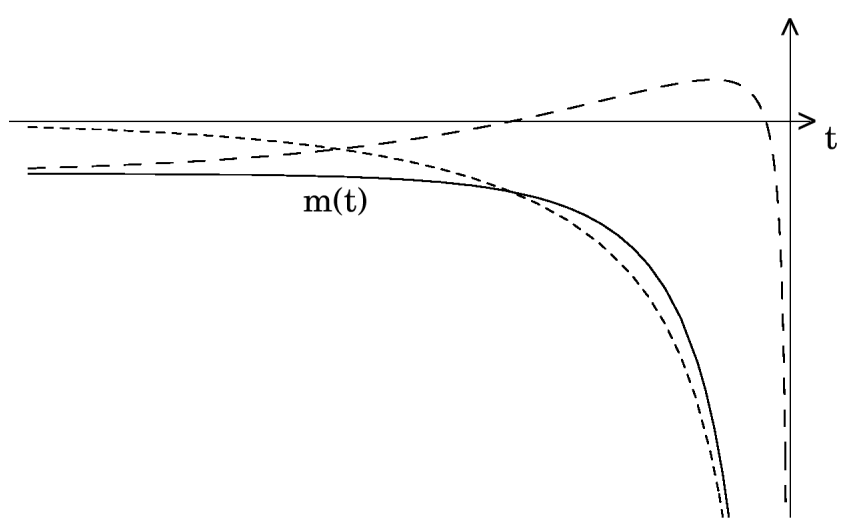

FIG. 1: The function $m(t)$ for $n=7$ is compared to the sqare root of the right hand side of relation (15), in small dashes. The difference is plotted in wide dashes and is positive in the interval of acceleration.

decelerating both as $\eta \rightarrow 0$ (corresponding to $t \rightarrow-\infty$ ) and as $\eta \rightarrow \infty$ (corresponding to $t \rightarrow 0$ from $t<0$ ). Specifically, one finds that

$$
S \sim \eta^{1 / 3} \quad(\eta \rightarrow 0)
$$

which corresponds to the 'stiff matter' equation of state $p=\rho$ (for pressure $p$ and mass density $\rho$ ), and

$$
S \sim\left(\eta-\eta_{0}\right)^{n /(n+2)} \quad(\eta \rightarrow \infty)
$$

which corresponds to the equation of state $p=-\left[\frac{n-4}{3 n}\right] \rho$; for $n=4$ this implies that the universe ends as a dustfilled Einstein de-Sitter universe, but for the $n=6,7$ cases of relevance to M-theory compactifications the final epoch is one with negative pressure matter, although the pressure is not sufficiently negative for acceleration. These two decelerating epochs are joined by an epoch of accelerated expansion, as shown in figure 2 for the $n=7$ case. The 'matter' responsible for this behaviour is of course the four-dimensional scalar field arising from the Kaluza-Klein mode that scales the volume of the compact internal space.

From these results one can see that the singularity of the function $K$ at $t=0$ is not a singularity of the Einstein-frame four-metric because $t=0$ is at an infinite proper time in the future of any event with $t<0$. It is also at an infinite proper time in the past of any event

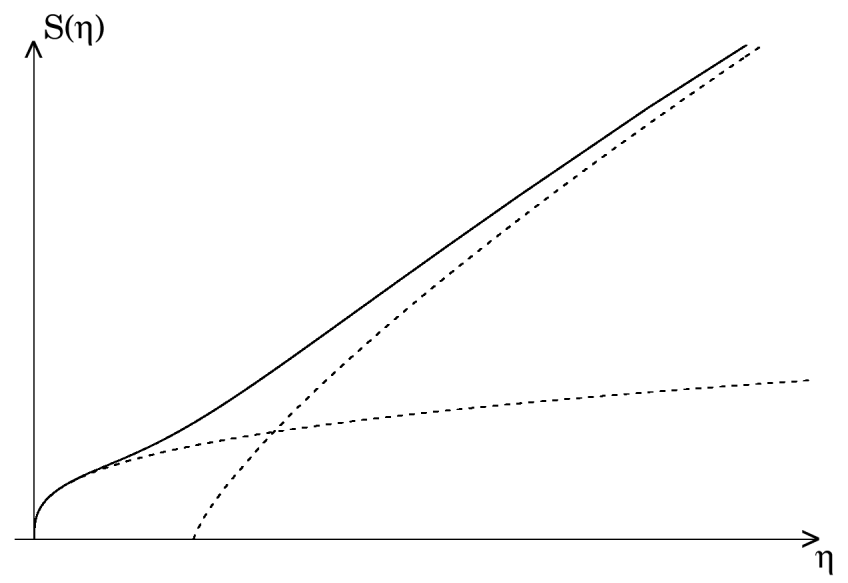

FIG. 2: The scale factor $S(\eta)$ of the four-dimensional universe is shown for $n=7$ and $\kappa=1$. It clearly exhibits an accelerating phase.

with $t>0$, so our solution really describes two possible universes, one for $t<0$ and another for $t>0$. Although the function $K$ is symmetric under $t \rightarrow-t$, the $(4+\mathrm{n})$ metric (9) is not, and only the $t<0$ case leads to a universe with an accelerating phase. Note that the $t<0$ and $t>0$ universes need not be (and are not) isometric; because $t=0$ is not in the spacetime, $t \rightarrow-t$ (in contrast to $\eta \rightarrow-\eta$ ) is not a transformation that reverses the cosmological flow.

We have now shown how the no-go theorem of [4, [5] may be circumvented by compactification on spaces with time-dependent metric. Such compactifications are, of course, typical of Kaluza-Klein cosmology, and have been extensively studied. However, no previous accelerating Kaluza-Klein cosmology that we are aware of can be considered as an escape from the no-go theorem of [4, [5], either because the strong energy condition is violated already in the higher dimension, or because the fourdimensional conformal frame is non-Einstein. Our solution has none of these undesirable features and yet not only has an accelerating phase, but also has a built-in mechanism to both start and stop acceleration.

Time-dependence of the internal metric was not in itself sufficient to yield an accelerating universe in four dimensions in Einstein frame. A hyperbolic compact internal space was also needed (because the analogous solution of the vacuum Einstein equations for an internal manifold of positive curvature does not allow acceleration). The absence of massless Kaluza-Klein vector fields in hyperbolic compactifications would be a serious defect in a traditional Kaluza-Klein cosmology, but is an advantage from the modern perspective in which matter lives on space-filling branes. This also allows the matter to be supersymmetric and suggests a possible mechanism for supersymmetry breaking by coupling to the non-supersymmetric 'bulk' gravitational theory arising 
from the supersymmetry breaking compactification (note that field theories with rigid supersymmetry can be coupled consistently, albeit non-supersymmetrically, to pure gravity).

A discussion of the cosmological advantages of hyperbolic compactifications can be found in [9, 10]. One such advantage, emphasized in [9] (where the relevant references to the mathematical literature may be found), arises from the remarkable fact that the only modulus of a compact hyperbolic Einstein space of dimension $n \geq 3$ is its volume, so only the volume can be time-dependent; this means that there is no 'rolling moduli' problem with this type of compactification. It seems remarkable that a model with so many attractive features can arise from a very simple compactification of M-theory.

Note added: After having sent an earlier version of this paper to the archives we learned that the solution of the vacuum Einstein equations found here is the 'zeroflux limit' of solutions of the non-vacuum Einstein equations obtained earlier in a different context by Chen et al. 11 and Ohta 12]. This point has since been elaborated on in a number of papers [13, 14, 15, 16, 17]. In the presence of flux, the relevant equations are not the vacuum Einstein equations and acceleration can also occur for compactification on spaces of non-negative curvature.

Acknowledgments. We are grateful to Gary Gibbons and Neil Turok for helpful discussions. We also thank Ishwaree Neupane for e-mail correspondence, which led us to make some minor clarifications. MNRW acknowledges financial support from the Gates Cambridge Trust.

* Electronic address: P.K.Townsend@damtp.cam.ac.uk

$\dagger$ Electronic address: M.N.R.Wohlfarth@damtp.cam.ac.uk

[1] A.G. Riess et al., The farthest known supernova: support for an accelerating universe and a glimpse of the epoch of deceleration, Astrophys. J. 560, 49 (2001).
[2] A. Lewis and S. Bridle, Cosmological parameters from CMB and other data: a Monte-Carlo approach, Phys. Rev. D66, 103511 (2002).

[3] C.L. Bennett et al., First year Wilkinson Microwave Anisotropy Probe (WMAP) observations: preliminary maps and basic results, astro-ph/0302207.

[4] G.W. Gibbons, Aspects of supergravity theories in Supersymmetry, Supergravity and Related Topics, edited by F. de Aguila, J.A. de Azcárraga and L. Ibañez, 346 (World Scientific, Singapore, 1985).

[5] J. Maldacena and C. Nuñez, Supergravity description of field theories on curved manifolds, and a no go theorem, Int. J. Mod. Phys. A16, 822 (2001).

[6] C.M. Hull and N.P. Warner, Non-compact gaugings from higher dimensions, Class. Quant. Grav. 5, 1517 (1988).

[7] P.K. Townsend, Quintessence from M-theory, JHEP 0111, 042 (2001).

[8] G.W. Gibbons and C.M. Hull, De Sitter space from warped supergravity solutions, hep-th/0111072.

[9] N. Kaloper, J. March-Russell, G.D. Starkman, and M. Trodden, Compact hyperbolic extra dimensions: branes, Kaluza-Klein modes and cosmology, Phys. Rev. Lett. 85, 928 (2000).

[10] G.D. Starkman, D. Stojkovic and M. Trodden, Large extra dimensions and cosmological problems, Phys. Rev. D63, 103511 (2001); Homogeneity, flatness and large extra dimensions, Phys. Rev. Lett. 87, 231303 (2001).

[11] C.-M. Chen, D.V. Gal'tsov and M. Gutperle, S-brane solutions in supergravity theories, Phys. Rev. D66, 024043 (2002).

[12] N. Ohta, Intersection rules for S-branes, Phys. Lett. B558, 213 (2003).

[13] N. Ohta, Accelerating cosmologies from S-branes, hep-th/0303238; A study of accelerating cosmologies from superstring/M theories, hep-th/0304172.

[14] S. Roy, Accelerating cosmologies from $\mathrm{M} /$ string theory compactifications, hep-th/0304084.

[15] M.N.R. Wohlfarth, Accelerating cosmologies and a phase transition in M-theory, hep-th/0304089.

[16] R. Emparan and J. Garriga, A note on accelerating cosmologies from compactifications and S-branes, hep-th/0304124.

[17] C.-M. Chen, P.-M. Ho, I.P. Neupane and J.E. Wang, A note on acceleration from product space compactification, hep-th/0304177. 